\title{
Analytic approximations for transit light curve observables and uncertainties
}

\author{
Joshua A. Carter ${ }^{1}$, Jennifer C. Yee ${ }^{2}$, Jason Eastman ${ }^{2}$, \\ B. Scott Gaudi ${ }^{2}$, Joshua N. Winn ${ }^{1}$ \\ ${ }^{1}$ Department of Physics, and Kavli Institute for Astrophysics and Space Research, \\ Massachusetts Institute of Technology, Cambridge, MA 02139 \\ ${ }^{2}$ Department of Astronomy, Ohio State University, 140 W. 18th Ave., Columbus, OH 43210
}

\begin{abstract}
The light curve of an exoplanetary transit can be used to estimate the planetary radius and other parameters of interest. Because accurate parameter estimation is a non-analytic and computationally intensive problem, it is often useful to have analytic approximations for the parameters as well as their uncertainties and covariances. Here we give such formulas, for the case of an exoplanet transiting a star with a uniform brightness distribution. When limb darkening is significant, our parameter sets are still useful, although our analytic formulas underpredict the covariances and uncertainties.
\end{abstract}

In general, the parameters of a transiting system and their uncertainties must be estimated from the photometric data using numerical methods. However, even when numerical algorithms are required for precise answers, it is often useful to have analytic approximations for the parameters as well as their uncertainties and covariances. Analytic approximations can be useful for planning observations, understanding the parameter degeneracies inherent in the model, calculating order-of-magnitude estimates of the observability of subtle transit effects, and designing low-correlation parameter sets that will speed the convergence of optimization algorithms.

To perform analytic calculations, we use a piecewise-linear approximation to the exact transit light curve, parameterized by $\left\{t_{c}, \tau, T, \delta, f_{0}\right\}$ as illustrated in Figure 1 (see also Seager \& Mallen-Ornelas 2003). The parameters are the midtransit time $\left(t_{c}\right)$, the out-oftransit flux $\left(f_{0}\right)$, the flux decrement during the full phase of the transit $(\delta)$, the duration of ingress or egress $(\tau)$, and the duration between the midpoint of ingress and the midpoint of egress $(T)$. For exact definitions of these parameters in terms of physical parameters (the impact parameter, scaled semimajor axis, and radius ratio), see Carter et al. (2008).

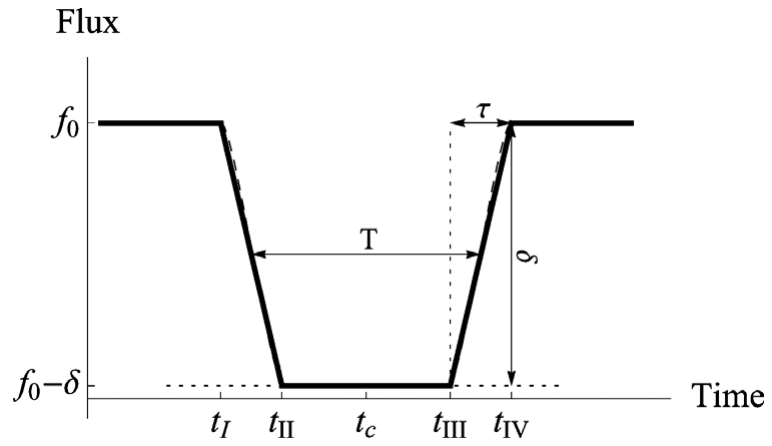

Figure 1. Linear model for a transit light curve with negligible limb darkening. Dashed line shows the physical light curve approximated by the linear model. 
Given the parameters $\left\{t_{c}, \tau, T, \delta, f_{0}\right\}$, it is possible to estimate the covariance matrix of the parameters that could be derived from observations of a light curve with some specified number of points $N$ and photometric errors $\sigma$ (see, e.g., Gould 2003). The accuracy of the results depends on the validity of the piecewise-linear model, and the assumptions that the photometric data points are evenly and densely spaced in time with independent Gaussian errors. Applying this technique (often referred to as Fisher information analysis), we obtain some simple and reasonably accurate expressions for the case in which the planet is small, the out-of-transit flux is known precisely, and limb darkening is negligible:

$$
\begin{aligned}
\sigma_{t_{c}} & =Q^{-1} T \sqrt{\tau / 2 T}, \\
\sigma_{\tau} & \approx Q^{-1} T \sqrt{6 \tau / T}, \\
\sigma_{T} & \approx Q^{-1} T \sqrt{2 \tau / T}, \\
\sigma_{\delta} & \approx Q^{-1} \delta .
\end{aligned}
$$

In these expressions, $Q \equiv \sqrt{N} \delta / \sigma$, the total signal-to-noise ratio of the transit in the small-planet limit. Using standard covariance propagation, we can estimate variances in other observables such as the mean stellar density in this same limit:

$$
\sigma_{\rho_{\star}}=Q^{-1} \rho_{\star} \sqrt{3 T / 8 \tau}
$$

For the complete covariance matrix, including the more general case in which the planet is not necessarly small and the out-of-transit flux is not known precisely, see Carter et al. (2008). That work also gives analytic formulas for other interesting "derived" quantities besides $\rho_{\star}$, and investigates the degree to which these formulas underpredict the errors and covariances when limb-darkening is appreciable. Complementary to this work is the numerical Fisher analysis for the case of a limb-darkened star recently presented by Pál (2008).

Not only do the parameters $\left\{t_{c}, \tau, T, \delta, f_{0}\right\}$ yield simple analytic expressions for their uncertainties and covariances; they are also weakly correlated over the majority of the allowed parameter space. As such these parameters provide a simple analytic framework for weighing the statistical merits of other parameter choices. For numerical fitting codes, a desirable parameter set has low mutual correlations and easily specified a priori likelihood functions. For this purpose we advocate a parameter set consisting of the midtransit time, the out-of-transit flux, the ratio of planetary to stellar radii $\left(R_{p} / R_{\star}\right)$, the normalized impact parameter, and the duration between the midpoint of ingress and the midpoint of egress $(T)$. Refer to Carter et al. (2008) for analysis of this parameter set and additional parameter sets that are essentially uncorrelated.

\section{Acknowledgements}

We thank Philip Nutzman, Sara Seager and Paul Joss for providing helpful comments.

\section{References}

Carter, J. A., Yee, J. C., Eastman, J., Gaudi, B. S., \& Winn, J. N. 2008, ArXiv e-prints, arXiv:0805.0238

Gould, A. 2003, ArXiv e-prints, arXiv:0310577

Pál, A. 2008, ArXiv e-prints, arXiv:0805.2157

Seager, S. \& Mallén-Ornelas, G. 2003, ApJ, 585, 1038 